\title{
STEADY-STATE PREFERRED ORIENTATION OF ICE DEFORMED IN PLANE STRAIN AT $-1^{\circ} \mathrm{C}$
}

\author{
By C. J. L. Wilson and D. S. Russell-HeAD* \\ (School of Earth Sciences, University of Melbourne, Parkville, Victoria 3052, Australia)
}

\begin{abstract}
Specimens of polycrystalline ice were experimentally deformed in plane strain at a temperature of $-1{ }^{\circ} \mathrm{C}$, under a constant load and with shortening strains varying from 2 to $27 \%$. The random $c$-axis orientation of the initial ice aggregates changes continuously with increasing strain towards a steady-state, smallcircle containing two maxima lying about the shortening direction. The ice microstructure is sequentially transformed from undeformed grains to smaller, irregular, deformed grains containing recrystallized grains. Grain growth then occurs forming a coarse interlocking-grain aggregate. These microstructural changes are attributed to solid-state processes occurring during deformation. The previously deformed specimens were then annealed without a load at $-1^{\circ} \mathrm{C}$. Annealing resulted in a substantial grain-size coarsening. However, no $c$-axis preferredorientation difference developed between annealed and unannealed samples.
\end{abstract}

RÉsUME. Orientation préférentielle d'équilibre de la glace déformée sous une sollicitation plane $\dot{a}-l^{\circ} \mathrm{C}$. Des échantillons de glace polycristalline ont èté déformés expérimentalement sous une sollicitation plane à une température de $-1^{\circ} \mathrm{C}$, sous une charge constante, avec des déformations par raccourcissement allant de 2 à $27 \%$. L'orientation arbitraire des axes $c$ de l'agrégat initial de glace se modifie continuellement lorsque la contrainte augmente jusqu'à un état d'équilibre, un petit cercle comprenant deux maximum approximativement dans la direction du raccourcissement. La microstructure de la glace est progressivement transformée depuis des grains indéformés jusqu'à des grains déformés, plus petits, irréguliers avec des grains de recristallisation. Alors intervient une croissance des grains qui forme un agrégat grossier de grains entremêlés. Ces changements microstructuraux sont attribués à des processus intervenant à l'état solide pendant la déformation. Les échantillons déjà déformés furent alors entourés d'un anneau sans charge à $-1^{\circ} \mathrm{C}$. L'annelation provoqua un grossissement substantiel des grains mais il ne se développa pas d'orientation préférentielle des axes $c$ différente dans les échantillons avec ou sans annelation.

ZUSAMmenfassung. Stationär bevorzugte Orientierung von Eis, das bei $-1^{\circ} \mathrm{C}$ eben deformiert wird. Proben polykristallinen Eises wurden experimentell bei einer Temperatur von $-1{ }^{\circ} \mathrm{C}$ unter konstanter Last und mit Schrumpfspannungen zwischen 2 und $27 \%$ eben deformiert. Die ursprünglich zufällige Orientierung der $c$-Achsen verändert sich mit wachsender Spannung kontinuierlich gegen einen stationären Zustand, bei dem in einem Kleinkreis zwei Maxima etwa in der Schrumpfrichtung liegen. Die Mikrostruktur des Eises wird allmählich von nicht deformierten Körnern in kleinere, unregelmässig verformte Körner verwandelt, wobei auch rekristallisierte Körner auftreten. Dann tritt ein Kornwachstum ein, das ein grobes, ineinandergreifendes Korngebilde erzeugt. Dieser Wandel der Mikrostruktur ist Festkörperprozessen zuzuschreiben, die bei der Deformation eintreten. Die verformten Proben wurden dann ohne Last bei $-1^{\circ} \mathrm{C}$ verfestigt. Die Verfestigung führte zu einer wesentlichen Vergröberung der Korngrösse, jedoch zeigte sich kein Unterschied in der bevorzugten Richtung der $c$-Achsen zwischen verfestigten und unverfestigten Proben.

\section{INTRODUCTION}

This paper presents the results of an experimental study investigating the microstructures and patterns of $c$-axis preferred orientations produced by plastic deformation of an aggregate of randomly oriented polycrystalline ice. The experiments have been conducted in a plane-strain deformation apparatus at a constant temperature of $-1{ }^{\circ} \mathrm{C}$ and with the same initial load. Comparable plane-strain apparatuses have previously been used by Kamb (1972) and by Budd and Matsuda (1974) who undertook pure shear deformations at temperatures varying from 0 to $-10^{\circ} \mathrm{C}$. These other studies were not specifically concerned with progressive microfabric changes with increasing deformation.

The microstructures associated with the deformation of individual ice grains have been well documented by Steinemann (1954, 1958), Rigsby (1960), Gold (1963), and Wakahama (1964) in thin-section deformation studies. These authors have shown that deformation of an existing grain structure is associated with intracrystalline slip and is accompanied by the formation of undulose extinction, deformation bands, and subgrains. All these are features of a dynamic recovery process. Dynamic recrystallization has also been recorded (Steinemann, 1958;

\footnotetext{
* Present address: Faculty of Engineering, University of Melbourne, Parkville, Victoria 3052, Australia.
} 
Wakahama, 1964). This phenomenon, which involves the nucleation and growth of new grains particularly at grain boundaries (Rigsby, 1960), extensively modifies the pre-existing microstructure (Steinemann, 1954). When deformation is stopped the nuclei that have formed continue to grow (Steinemann, 1954; Wakahama, 1964), leading to rapid static recrystallization. The grains resulting from this static recrystallization are larger than the average grain resulting from dynamic recrystallization because the nuclei that would be produced by a further incremental strain do not form. In the case of both dynamic and static recrystallization, the dependence of grain-size, and hence microstructure, on temperature, strain-rate, and stress is similar, as pointed out by Rigsby (1960) and Wakahama (1962).

The role of intracrystalline slip processes in modifying $c$-axis preferred-orientation patterns have been observed by Wakahama (1964). Preferred-orientation measurements recorded in the majority of experimental studies on polycrystalline ice are from material where no separation, on microstructural grounds, has been made between deformed grains and dynamically or statically recrystallized grains. However, the uniaxial compression studies of Steinemann (1954) and subsequent workers commonly report a strong $45^{\circ}$ small-circle distribution of $c$-axes about the shortening direction.

\section{EXPERIMENTAL METHODS}

Two different types of experiments have been performed:

(1) Experiments in which a randomly oriented aggregate of ice is strained at $-1^{\circ} \mathrm{C}$ with a constant load of $1.5 \mathrm{kN}$ in a plane-strain apparatus. These will be referred to as plane-strain deformation experiments.

(2) Experiments in which a part of the deformed sample is then annealed at $-1^{\circ} \mathrm{C}$ without a load. They are referred to as annealing experiments.

The plane-strain experiments were performed in the apparatus illustrated in Figure 1. During shortening, samples were confined in the $Y$ direction and were unconstrained and free to extend in a direction $(X)$ normal to the shortening direction $(Z)$ (Fig. 1). The ice sample is fitted between two fixed "restraint" anvils and compressed by the load anvils. Lead bars are lowered onto the

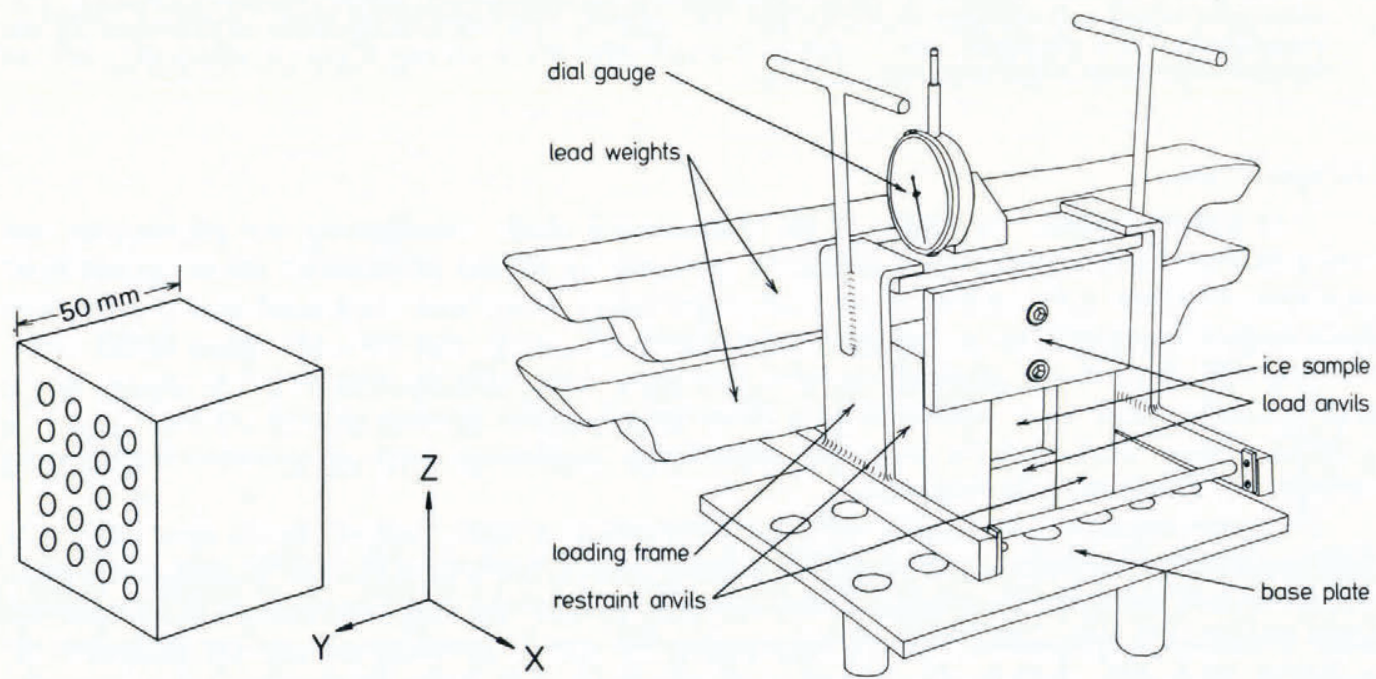

Fig. 1. Sketch of deformation apparatus and ice sample with circular strain markers. Weights on the viewer's side of the loading frame have been removed to reveal the sample under load. The axes of bulk strain are shown in relation to the sample and apparatus. 


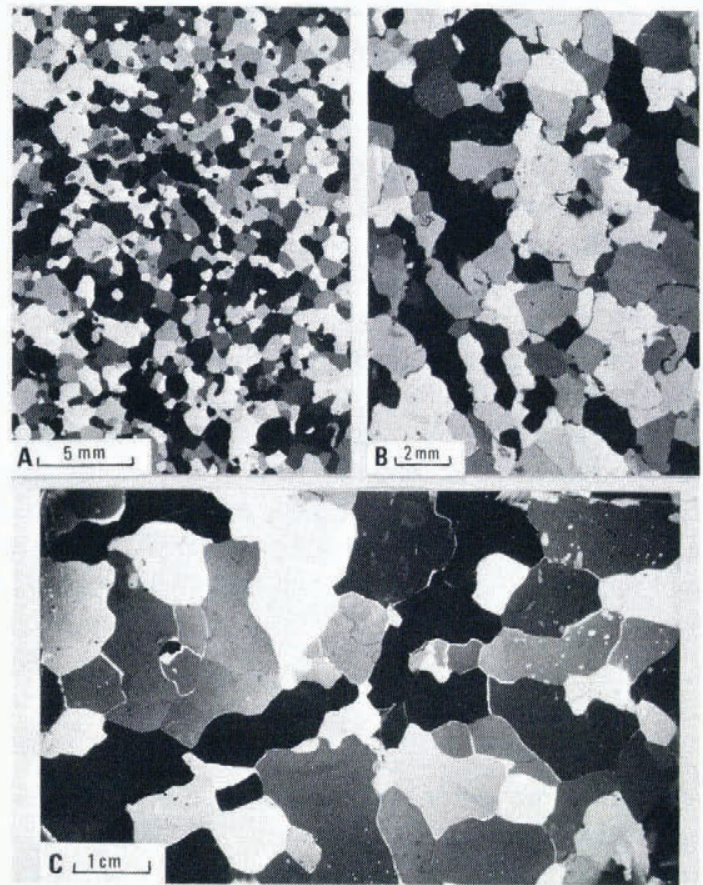

Fig. 2. Microstructures associated with the undeformed polycrystalline ice used as starting materials. A: Fine-grained ice used in experiments 6 to 10. B: Medium-grained ice used in experiments 1 to 5. C: Undeformed mediumgrained ice annealed at $-1^{\circ} \mathrm{C}$ for $17 \mathrm{~d}$.

loading frame which, in turn, bears upon the upper plate of the top anvil. Side plates allow the free vertical movement of the top anvil within the restraint anvils. The vertical displacement of the upper surface of the ice sample is indicaied by the dial gauge. The supporting base plate is mounted on three substantial legs and all the mild-steel components are nickel plated for corrosion protection and for smoothness of the faces that mate with the ice.

The apparatus is immersed in an insulated tank of circulated kerosene, and the whole placed within a domestic freezer chest set typically to $-22^{\circ} \mathrm{C}$. Heaters fitted under the base plate of the apparatus are turned on or off according to the balance state of a resistance bridge, one arm of which is a thermistor sensing the kerosene temperature. A measure of protection against sample loss in the event of a power failure is afforded by this arrangement.

The temperature of the kerosene during sample deformation was held at $-1.00 \pm 0.01^{\circ} \mathrm{C}$. During loading and unloading the temperature was allowed to fall typically to $-10^{\circ} \mathrm{C}$. By lowering the temperature after deformation and undertaking a slow quench of the ice, prior to unloading, we attempted to prevent static recrystallization and hence preserve the microstructure achieved during the deformation. The success of this could not be verified in the current set of experiments.

The ice used in this investigation was either fine-grained $(0.7 \mathrm{~mm})$ or medium-grained $(1.2 \mathrm{~mm})$ (Fig. 2). The polycrystalline ice was prepared from a mixture of sieved crushed ice and distilled water. The crushed ice was obtained from a single crystal of ice grown from deionized and deaerated distilled water. Air bubbles were mechanically removed from the ice-water mixture leaving an irregular distribution of small bubbles. Low concentrations of 0.1 to $0.2 \mathrm{~mm}$ diameter bubbles were confined to grain boundaries. The fine-grained ice sometimes contained bubble concentrations up to $10 \mathrm{~mm}^{-3}$. Excess distilled water was removed by lightly compressing the sample prior to freezing. Before the polycrystalline ice was used, it was annealed 
for periods varying from two weeks to five months at a temperature of $-10^{\circ} \mathrm{C}$. The microstructure of the resulting polycrystalline ice consists of an interlocking grain aggregate with irregular serrated grain boundaries showing no undulatory extinction (Fig. 2).

Rectangular blocks of polycrystalline ice were cut approximately to size and shape on a band-saw. The final dimensions of $45 \mathrm{~mm} \times 50 \mathrm{~mm} \times 62 \mathrm{~mm}$ were achieved by grinding on different grades of carborundum paper. In most samples strain markers were inscribed on the $X Z$ faces adjacent to the constraining anvils (Fig. 1). These strain markers were circular grooves $5 \mathrm{~mm}$ in diameter, $0.4 \mathrm{~mm}$ deep, and $0.3 \mathrm{~mm}$ wide, inscribed on $10 \mathrm{~mm}$ centres. The positioning of these markers was aided by an acrylic templet. The majority of the grooves was left unfilled and contained only air. Others were filled with diluted Indian ink. The latter markers were of no value as they were unrecognizable after deformation at temperatures of $-1^{\circ} \mathrm{C}$. The initial stress was approximately $0.6 \mathrm{MPa}$ axial. However, during an experiment there was a change in crosssectional area and a stress reduction as shown in Table I. Examples of experimental results are presented in Figure 3. Thin sectioning, photography, and preferred-orientation measurements were undertaken in a cold room at $-10^{\circ} \mathrm{C}$. Preferred orientations were obtained using Leitz and Rigsby universal stages and data have been plotted as scatter diagrams on the lower hemisphere of an equal-area net.

\section{STRAIN HOMOGENEITY}

The blocks of polycrystalline ice deformed homogeneously on a scale greater than a few grain diameters. Numerous small-amplitude undulations (parallel to $Y$ ) formed on the $Y Z$ faces of both the fine- and the medium-grained samples. They had amplitudes and wavelengths of the

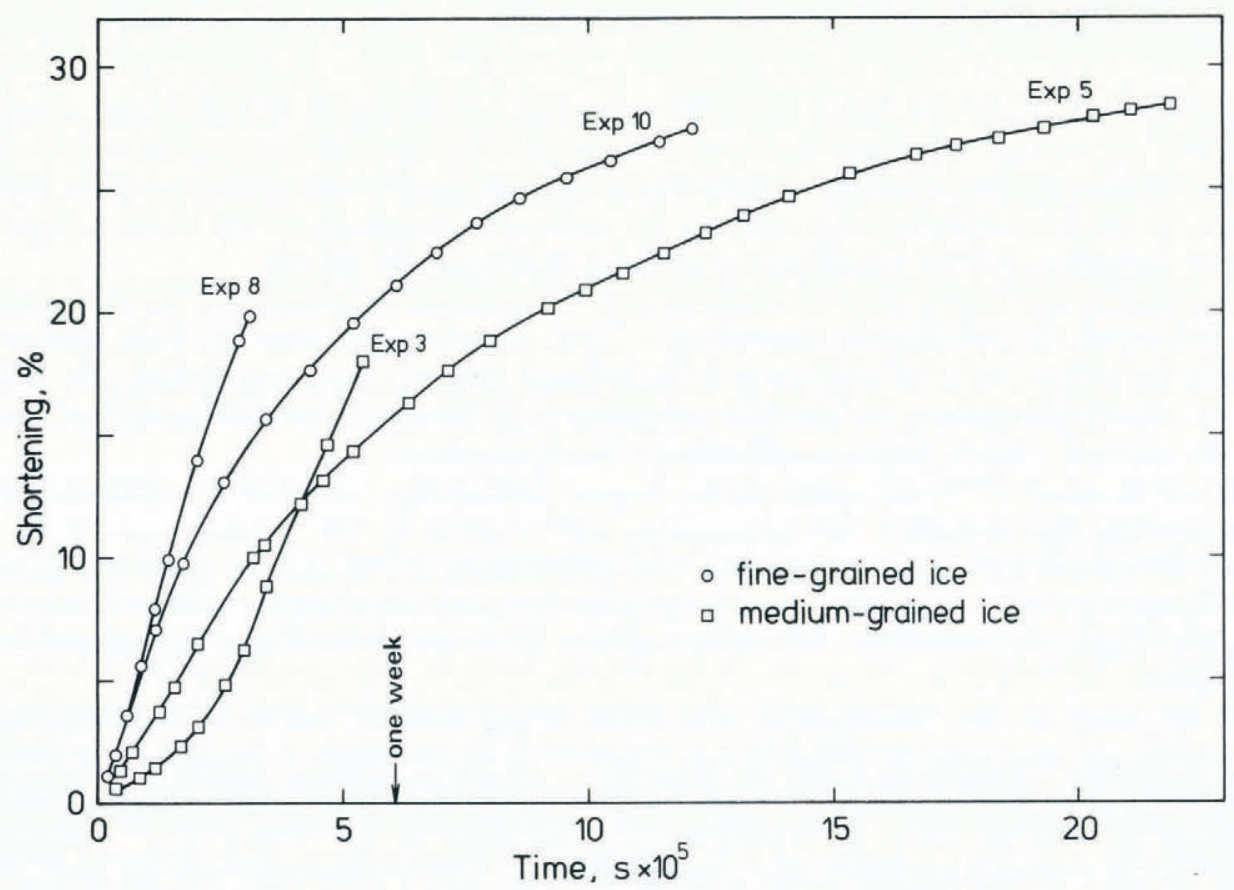

Fig. 3. Shortening plotted against time for some of the plane-strain samples. The variability of strain histories is due mainly to (a) differences in the initial exactness of fit of the ice samples between the anvils, and (b) frictional effects that are not reproducible from one experiment to the next. 
TABLE I. SUMMARY OF EXPERIMENTS

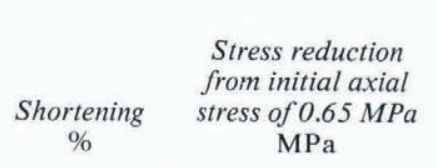

Mean grain size $\bar{d}$
of deformed sample
measured on a Rigsby
stage
$\mathrm{mm}$
Mean grain size $\bar{d}$ of annealed sample $\mathrm{mm}$

Number of
days sample
annealed

Medium

Starting

$\overline{0.05}$

0.07

0.12

0.13

0.19 material

Experiment 1

Experiment 2

Experiment 3

Experiment 4

Experiment 5

-2
7
19
22
27

Fine

Starting

material

Experiment 6

Experiment 7

Experiment 8

Experiment 9

Experiment 10

$\begin{array}{rr}- & -\overline{01} \\ 5 & 0.03 \\ 9 & 0.07 \\ 19 & 0.12 \\ 27 & 0.19\end{array}$

1.1 to 1.3
1.1
1.12
1.02
1.5
2.4

$\begin{array}{lr}4.0 & 42 \\ 3.07 & 39 \\ 2.5 & 17 \\ 3.2 & 17 \\ 7.00 & 42 \\ \text { NOT ANNEALED }\end{array}$

$\begin{array}{ll}1.8 & 20 \\ 2.6 & 39 \\ 3.5 & 39 \\ & \\ 2.5 & 15 \\ 2.2 & 39 \\ 3.3 & 19\end{array}$

order of the average grain-size. There is no evidence to suggest that zones of heterogeneous deformation existed, or that the anvil wall perpendicular to the $Y$ axis retarded the extension in the $X$ direction.

The circular strain markers on the plane perpendicular to the $Y$ axis deformed into a set of ellipses that yield individual $x / y$ and $z / y$ ratios. Because $y$ is unchanged from the initial dimension, these values are the elongation recorded in the $X$ direction and the shortening in the $Z$ direction (Fig. 4). In most experiments there was a uniform distortion of the markers into a set of similar ellipses suggesting that the extension in the $X$ direction in the $X Z$ plane was uniform. In experiments 6 and 7 (Fig. 4A), however, the $x / z$ ratios near the base were marginally greater than those observed in the top half of the deformed specimen. The large bubbles in both the fineand medium-grained ice were also elongated parallel to the major axis of the strain ellipses. They were prolate ellipsoids, having $x / z$ ratios comparable to those of the adjacent strain ellipses.

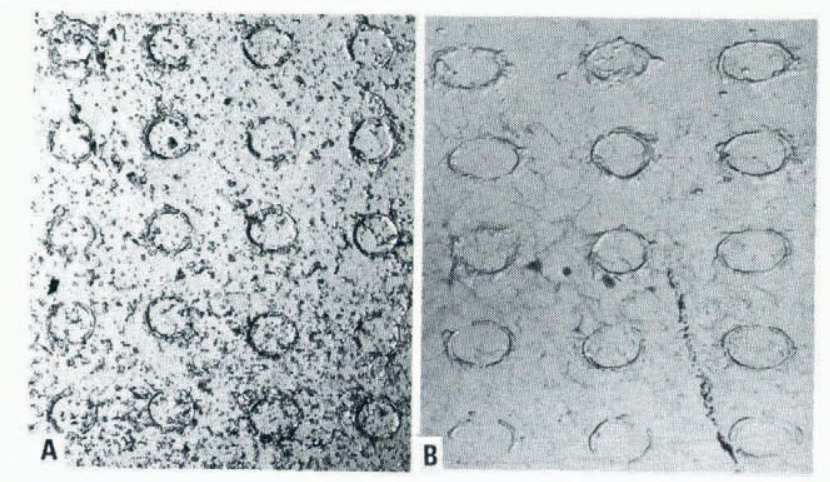

Fig. 4. Thick sections containing strain markers in deformed ice. The initial markers were circles with diameters of $5 \mathrm{~mm}$. A: Experiment 7 (5\% shortening) illustrates a subtle difference between the $x / z$ ratios recognized in the upper half and the more deformed lower half. A higher concentration of air bubbles can also be noted in the finegrained ice used in experiment 7. B: Experiment 3 (19\% shortening) illustrates a uniform distribution of strain. 


\section{GRAIN-SIZE VARIATION}

The size $\bar{d}$ of the polycrystalline ice grains studied in this investigation has been measured (Table I) in optical sections using the mean linear intercept (m.l.i.) method. The m.l.i. is measured from a linear traverse by counting the number $N$ of grain boundaries or grains which intercept a linear traverse of length $L$. The m.l.i. is given by:

$$
\text { m.l.i. }=\bar{d}=\frac{L}{N}=\frac{1}{N_{\mathrm{L}}}
$$

where $N$ is the number of grain boundaries or grains and $N_{\mathrm{L}}$ the number of grain boundaries or grains per unit length of traverse.

However, as pointed out by Exner (1972) and Pickering (1976), this technique does not give a true size distribution as corrections have to be made for overlapping and three-dimensional effects. $\bar{d}$ is generally less than the average diameter of the grains comprising the microstructure $\bar{D}$, which, in turn, is less than the maximum grain diameter $D_{\mathrm{M}}$. The relationship between $\bar{d}, \bar{D}$, and $D_{\mathrm{M}}$ depends on the grain shape assumed. The majority of the ice grains were equidimensional in most sections measured. Therefore the mean diameter $\bar{D}$ of the grain aggregate is (Pickering, 1976)

$$
\bar{D} \approx 1.75 \bar{d} \text {. }
$$

In the case of the annealing experiments 9 and 10, the grain-size of the polycrystalline ice was measured from photomicrographs as the specimens and thin sections were destroyed in a cold-room failure. The results were compatible with those of the other experiments. However, using the m.l.i. method on photographs tended to give a higher value for $\bar{d}$ than if the original thin section was used. This is because many grain boundaries or grains could not be discerned on the photograph. At least eight m.l.i. traverses of each sample were made in polarized light, and the number of grains $N$ recognized in a $20 \mathrm{~mm}$ or $50 \mathrm{~mm}$ graticule were recorded.

In the plane-strain deformation experiments there was no obvious change in the size $\bar{d}$ at $2 \%$ shortening in the fine-grained ice, and up to $7 \%$ shortening in the medium-grained ice. At $5 \%$ and $19 \%$ shortenings in the fine- and medium-grained ice aggregates, respectively, there was a notable grain-size reduction. At higher shortenings grain sizes increased progressively. Except for experiments 6 and 7 there was a homogeneous distribution of grain sizes throughout a sample.

In experiment 6 , the deformed sample was $0.3 \%$ wider at the base than at the top. The lower portion of the specimen was more deformed than the upper, with the bulk strain ( $2 \%$ shortening) grading uniformly from the base to the top. In a vertical $X Z$ section this strain variation was reflected by a grain-size change. The upper portion of the sample had a grain-size identical to that of the starting material $(\bar{d}=0.74)$, whereas the lower portion had a grain-size of 0.66 . However, microscopic observations suggested there was another grain population with $\bar{d}=0.3 \mathrm{~mm}$. The deformation in experiment 7 was also inhomogeneous with the sample being $2.6 \%$ wider at the base than at the top. The value of $\bar{d}$ was uniform throughout the $X Z$ section (0.46), but was smaller than that observed in an $X Y$ section (0.5) taken from the top of the specimen. Observation of this sample under an optical microscope indicated that there was another grain population $(\bar{d}=0.3 \mathrm{~mm})$. These finer grains also had a uniform size and were evenly distributed throughout the sample in an $X Z$ section.

Annealing produced an increase in grain-size for all the samples investigated. As the annealing experiments were of different durations, a quantitative correlation of grain-size increase with annealing time is difficult. The small grain-size observed in the plane-strain deformation experiments on the fine-grained samples, is retained after undertaking the annealing experiments. There was a uniformity of grain-sizes in all experiments except experiment 3 , where there were irregular regions of fine-grained ice within coarse. The grain size-distribution in the 
starting materials (Fig. 3c) was also non-uniform, particularly in the annealed medium-grained ice.

\section{c-AXIS ORIENTATION PATTERNS}

\section{Starting material}

The $c$-axis patterns (Fig. 5) were weak with small pole-free areas, low symmetry, and low maximum concentrations. They were also non-reproducible between and within specimens and often had the characteristics of a truly random $c$-axis pattern (Wilson, 1973). Annealing the medium-grained starting material for $17 \mathrm{~d}$ at $-1^{\circ} \mathrm{C}$ results in an increase in grain size from $\bar{d}=0.20$ to $\bar{d}=1.35$. However, after annealing the basic $c$-axis orientation pattern remained unchanged (Fig. 5C and D) and no reproducibility was obtained when separate sections were measured from the same specimen and compared.

\section{Plane-strain deformation of medium-grained ice}

A sequence of progressive plane-strain experiments using the randomly-oriented mediumgrained ice developed the orientation patterns shown in Figure 6. After $2 \%$ shortening (Experiment 1) there is only a subtle change from the random orientation observed in the starting material. There appears to be a decrease in the number of grains with $c$-axes lying in the $X Y$ plane. After $8 \%$ shortening (Experiment 2) there is a marked change in the $c$-axis pattern to two irregular concentrations distributed between the 20 and $65^{\circ}$ small-circle cones centred about the shortening axis $Z$. There is an absence of $c$-axes about the extension axis $X$. These patterns can be observed in both the $X Z$ or $X Y$ sections.

In experiments 3 and 4 (shortenings of 19 and $22 \%$ respectively) the $c$-axis patterns are contained between the 25 and $45^{\circ}$ small-circle cones centred about $Z$. In experiment 3 they are concentrated into two maxima that subtend an angle of approximately $120^{\circ}$, whereas in experiment 4 this pattern is more diffuse.
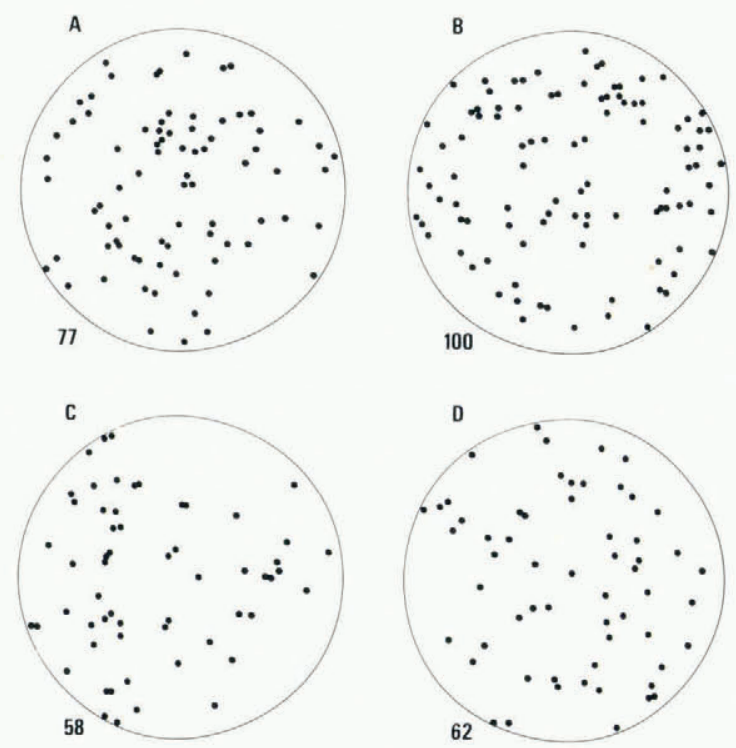

Fig. 5. Representative c-axes patterns for ice used as starting material. A: medium-grained ice used in experiment 4. B: fine-grained ice used in experiment 7. $C:$ annealed medium-grained materiäl used in experiment 4 , annealed at $-I^{\circ} \mathrm{C}$ for $17 \mathrm{~d}$. D: annealed fine-grained starting material used in experiment 7 , annealed at $-1^{\circ} \mathrm{C}$ for $39 \mathrm{~d}$. 

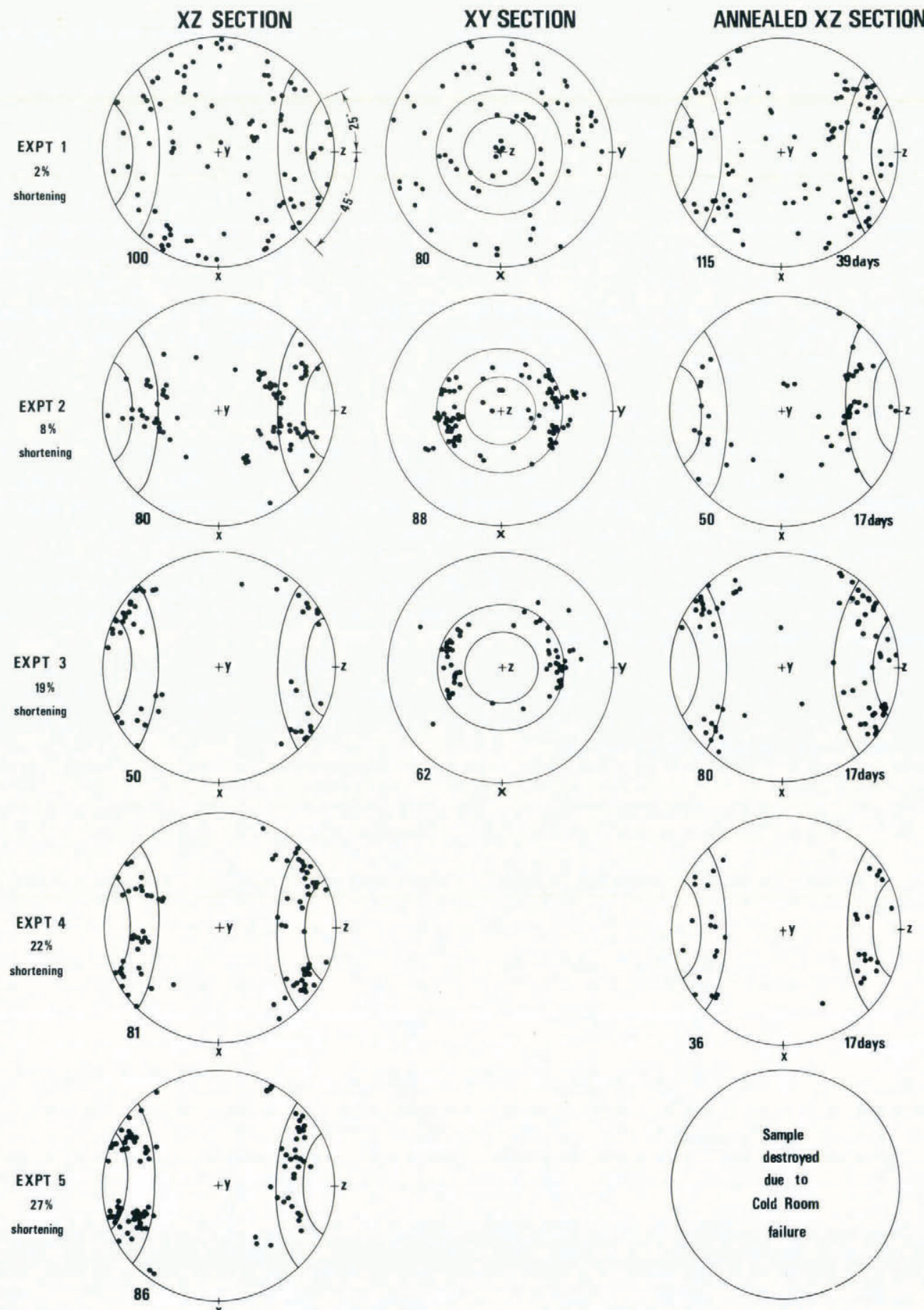

Fig. 6. Patterns of c-axis orientations observed in deformed medium-grained ice. $c$-axes were recorded from sections parallel to the $X Z$ plane in both the deformed and annealed samples in experiments 1 to 5 . In experiments 1 to 3 , sections were also prepared parallel to the $X Y$ plane. The superimposed small-circles are at $25^{\circ}$ and $45^{\circ}$ to the shortening axis $Z$. The number of c-axes is shown on the bottom left of each projection and the number of days any sample was annealed is shown on the bottom right of the projection. 
Experiment 5 (27\% shortening) produced two distinct $c$-axis maxima. These subtend an angle of approximately $90^{\circ}$ and lie between the two small-circle cones with opening angles of $30^{\circ}$ to $45^{\circ}$ about $Z$. There was a notable absence of $c$-axes oriented in the $X Y$ plane or parallel to the shortening axis $Z$.

\section{Plane-strain deformation of fine-grained ice}

Experiments 6 to 10 (Fig. 7) illustrate the sequence of development of the $c$-axis preferred orientation in the fine-grained ice. No preferred-orientation data are presented for experiment 8

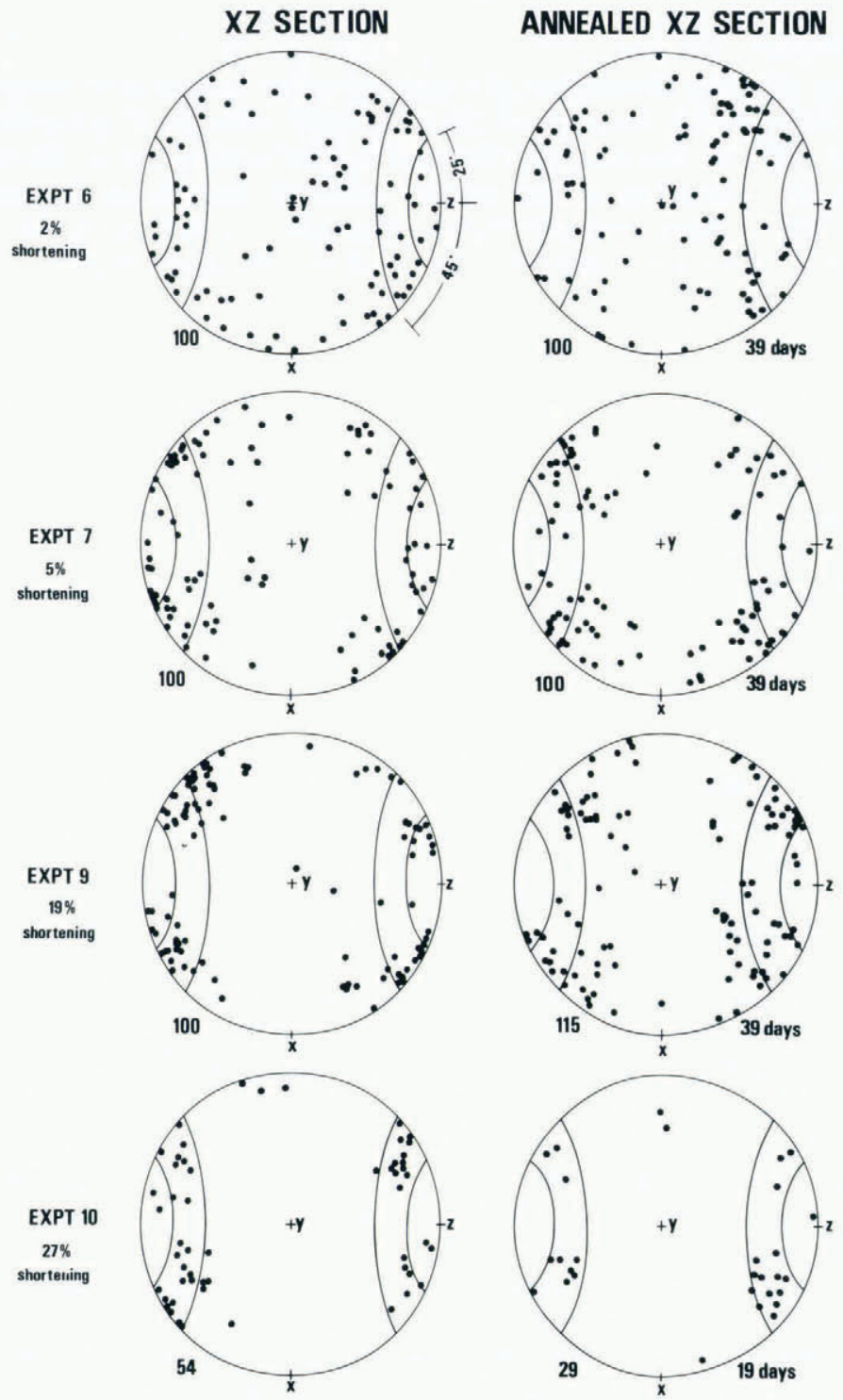

Fig. 7. Patterns of c-axis orientations observed in deformed fine-grained ice. All measurements were recorded from $X Z$ sections. The superimposed small circles are at $25^{\circ}$ and $45^{\circ}$ to the shortening axis $Z$. The number of $c$-axes is shown on the bottom left of each projection and the number of days any sample was annealed is shown on the bottom right of the projection. 
as the sample and thin sections melted during a freezer malfunction before sufficient data could be recorded. After $2 \%$ shortening (Experiment 6 ) there is no obvious change from the $c$-axis patterns observed in the starting material. At 5\% shortening (Experiment 7 ) the $c$-axes are distributed as two diffuse maxima between $Z$ and the $60^{\circ}$ small-circle cone. As a result of $19 \%$ and $27 \%$ shortening (Experiments 8 and 10) two $c$-axis concentrations are developed between the 20 and $45^{\circ}$ small-circle cones centred on $Z$, and subtend angles of 120 and $90^{\circ}$ respectively. The change in $c$-axis patterns from $5 \%$ to $27 \%$ is therefore marked by the development of two concentrations in a discrete small-circle about $Z$ and an absence of grains with $c$-axes lying parallel to $Z$ or straddling the $X Z$ plane.

\section{Annealing experiments}

In all annealed samples the number of $c$-axes per given section area measured was smaller than for the parent material, because of the substantial increase in grain-size. The annealed samples produced patterns of $c$-axis orientation that were comparable to those observed in the unannealed material. Annealing of the starting material reproduced the original randomly oriented pattern. The features produced during progressive shortening of the medium- (Fig. 6) and fine-grained (Fig. 7) ice are strongly reflected in all the annealed samples.

\section{Microstructural VARIATION}

\section{Plane-strain deformation}

There are many microstructures common to the deformed samples and the difference between experimental sections is not pronounced. The main features observed with the progressive deformation are a change in grain size, an interlocking of grains, and the presence of recovery features such as deformation bands. These features are discussed in more detail below:

\section{Experiments 1 to 5}

Microstructures comparable to those of the starting material were observed at up to $7 \%$ shortening (Fig. 8A). Unlike the starting material, the deformed grains possess broad zones of undulose extinction. Grain boundaries are well defined, being straight or only slightly curved, and bubbles are often concentrated along these boundaries. Grains form an interlocking aggregate and grain-sizes in both the $X Y$ and $X Z$ planes are mostly equant, but with some elongate grains. However, the elongate grains do not protrude into the adjacent grains. There is a suggestion that grains are elongated in the direction parallel to the $X Y$ plane.

In experiment 3 (19\% shortening) the equant grain aggregate contains numerous well-defined deformation bands (Fig. 8B). Microscopic examination of this aggregate reveals other smaller equidimensional strain-free grains located at grain boundaries and within or adjacent to deformation bands (Fig. 8C and D). These smaller grains may occur individually (Fig. 8C) or as aggregates (Fig. 8D) and are responsible for the apparent reduction in grain-size observed in the sample (Table I). These grains appear to be a new generation of grains nucleated by a recrystallization process during deformation. They are not obvious if the thin section is observed only on the Rigsby stage.

Two distinct grain types exist in experiment 4 (22\% shortening), these are: (1) large grains that possess numerous deformation bands (Fig. 8E) and (2) small equant grains with curved grain boundaries that rarely have undulose extinction. The majority of the smaller grains in the $X Z$ section correspond to grains in which the $c$-axis lies between the 25 and $45^{\circ}$ small-circle cones (Fig. 6). Experiment 5 (Fig. 8F) is similar to experiment 4 except that the demarcation between grain types is not obvious and all grains possess deformation bands. The average grain- 

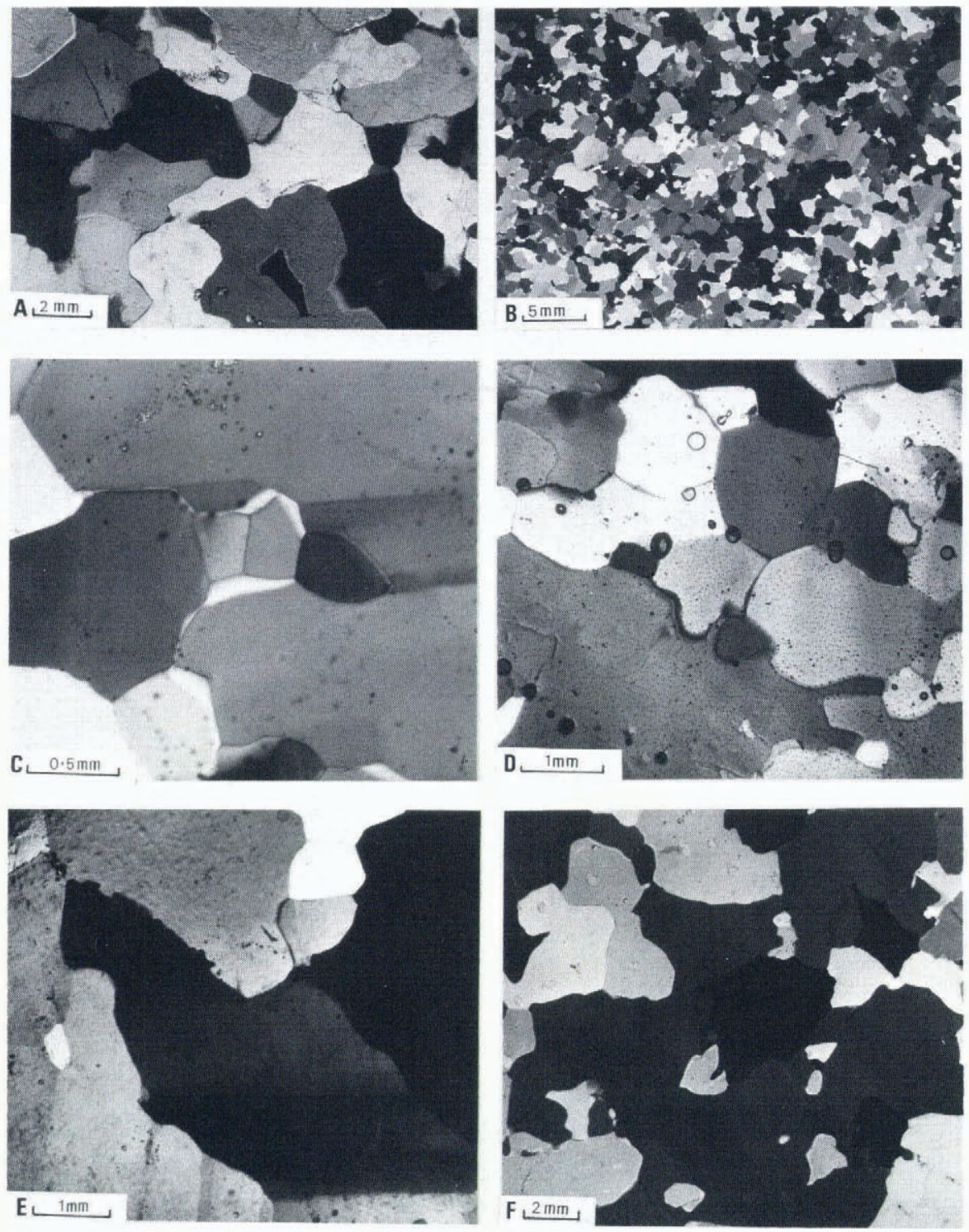

Fig. 8. Microstructures observed in XZ sections of deformed medium-grained ice experiments 1 to 5 . A: Experiment 2 after 7\% shortening. B: Experiment 3 after 19\% shortening illustrating the grain aggregate and the numerous deformation bands. C: Microphotograph from experiment 3 illustrating small new grains nucleated adjacent to a deformation band. D: Microphotograph from experiment 3 showing aggregate of small new grains. E: Microphotograph from experiment 4 illustrating large deformed grains with deformation bands that contain elongate concentrations of fine bubbles and small equant grains. F: The grain aggregate observed in experiment 5.

size is appreciably larger and grain boundaries are gently curved. Many grains contain trains of fine bubbles that are unrelated to the prevailing grain structure. The distribution of these bubbles suggests that they are relics from a pre-existing microstructure, such as that observed in experiment 4 . 
Experiments 6 to 10

Experiment 6 exhibits distinct grain-size and microstructural differences between the top and bottom of the specimen (Fig. 9A and B). The upper section (Fig. 9B) contains large irregularly shaped grains with continuous undulose extinction (up to $10^{\circ}$ ) across most grains. Grain
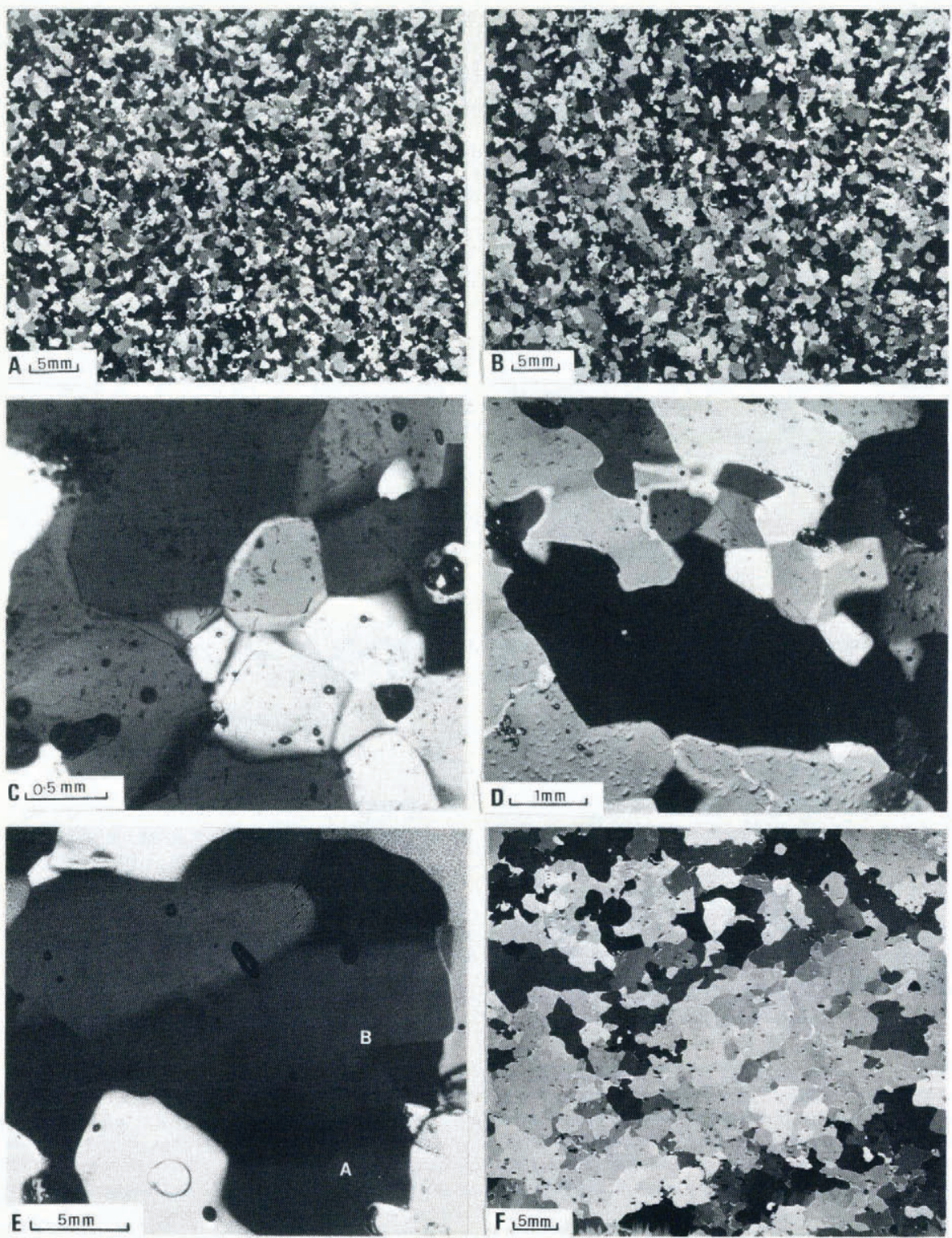

Fig. 9. Microstructures observed in deformed fine-grained ice in experiments 6 to 10. A: XZ section of experiment 6 after $2 \%$ shortening. The top (left-hand side of photograph) of deformed sample is coarser grained than the base. $B: X Y$ section of experiment 6 (2\% shortening) from upper portion of sample. $C$ : Microphotograph of XZ section of experiment 7 after 5\% shortening. D: Microphotograph of XZ section of experiment 8 after $9 \%$ shortening. E: Microphotograph of experiment 8 showing stepped deformation band at $A$ and terminated deformation band at $B . F: X Z$ section of experiment 10 after $27 \%$ shortening. 
boundaries are outlined by concentrations of fine bubbles in their margins (Fig. 9B). A few isolated small strain-free grains were observed on the margins of the large grains in the upper portion of the sample, whereas the lower half of the specimen contains two distinct grain types. Large irregular, undulose grains are rimmed by smaller rectangular or spherical grains or subgrains. Microstructures comparable to experiment 6 are also observed in experiment 7 (Fig. 9C). There is a ubiquitous subgrain structure, the greatest development being in the more deformed lower half of the sample.

After 9\% shortening (Experiment 8) a marked bi-modal grain structure is developed. It is characterized by the coexistence of large deformed grains and smaller undeformed grains (Fig. 9D). The grain boundaries of the large grains are defined by trains of fine bubbles, and internally these grains contain deformation bands (Fig. 9E). The deformation bands vary considerably in width and in the number contained within any grain. Individual bands may be continuous across a grain, terminated within a grain, or stepped. The deformation-band boundaries may also contain fine bubbles. Some of the small grains are larger than the smaller grains seen in experiment 7 (Fig. 9C) and are markedly misoriented (up to $30^{\circ}$ ) with respect to adjacent grains. Some of the small grains also contain wide, continuous deformation bands. Grain boundaries associated with the small grains are either planar or gently curved and only rarely contain a concentration of air bubbles.

In experiments 9 (Fig. 9F) and 10 there has been appreciable grain growth, resulting in an interlocking grain structure. There is no evidence for a bi-modal grain structure, there being a transition in sizes between large and smaller irregular grains, both of which contain deformation bands.

\section{Annealing experiments}

Annealing of the starting material results in the formation of strain-free, polygonal shaped, equant grains with smoothly curved or straight interfaces (Figs $3 \mathrm{C}$ and 10A). The shapes of these grains in any three orthogonal thin-sections are polygonal with dihedral angles of approximately $120^{\circ}$.

The annealing experiments undertaken with the deformed ice produced large increases in grain size and grain shape (Fig. 10). Grains exhibiting deformation-recovery features such as deformation bands were absent in all sections. All grains are bounded by smoothly curved grain boundaries, and the shape of the boundaries is consistent with the idea that they are migrating towards their centres of curvature. Grain shapes and sizes are irregular (Fig. 10B to G) and the polygonal microstructure achieved by annealing the starting material was not obtained in these experiments. The different grain-sizes recognized in the annealing experiments (e.g. Fig. 10B and C) appear to be, in part, a function of the period of anneal (Table I). However, in experiments 8 and 9 (Fig. 10F and $\mathrm{G}$ ) the inverse relationship exists, with smaller grains in the more-deformed and longer-annealed samples. Air bubbles are usually distributed within grains (Fig. 10E) and not on grain boundaries. The initial uniform distribution and high concentration of bubbles in the fine ice disappear on annealing with the development of larger and more dispersed bubbles (Fig. $10 \mathrm{~F}$ and $\mathrm{G})$.

\section{Discussion}

\section{Microstructural development}

At small strains the grain structure showed little obvious change in the average grain diameter. However, intracrystalline deformation features such as undulose extinction and deformation bands developed; there was no evidence for the existence of deformation lamellae or slip bands. The intracrystalline features observed in these experiments are analogous to dynamic recovery features described in the materials-science literature (McQueen and Jonas, 1975). 

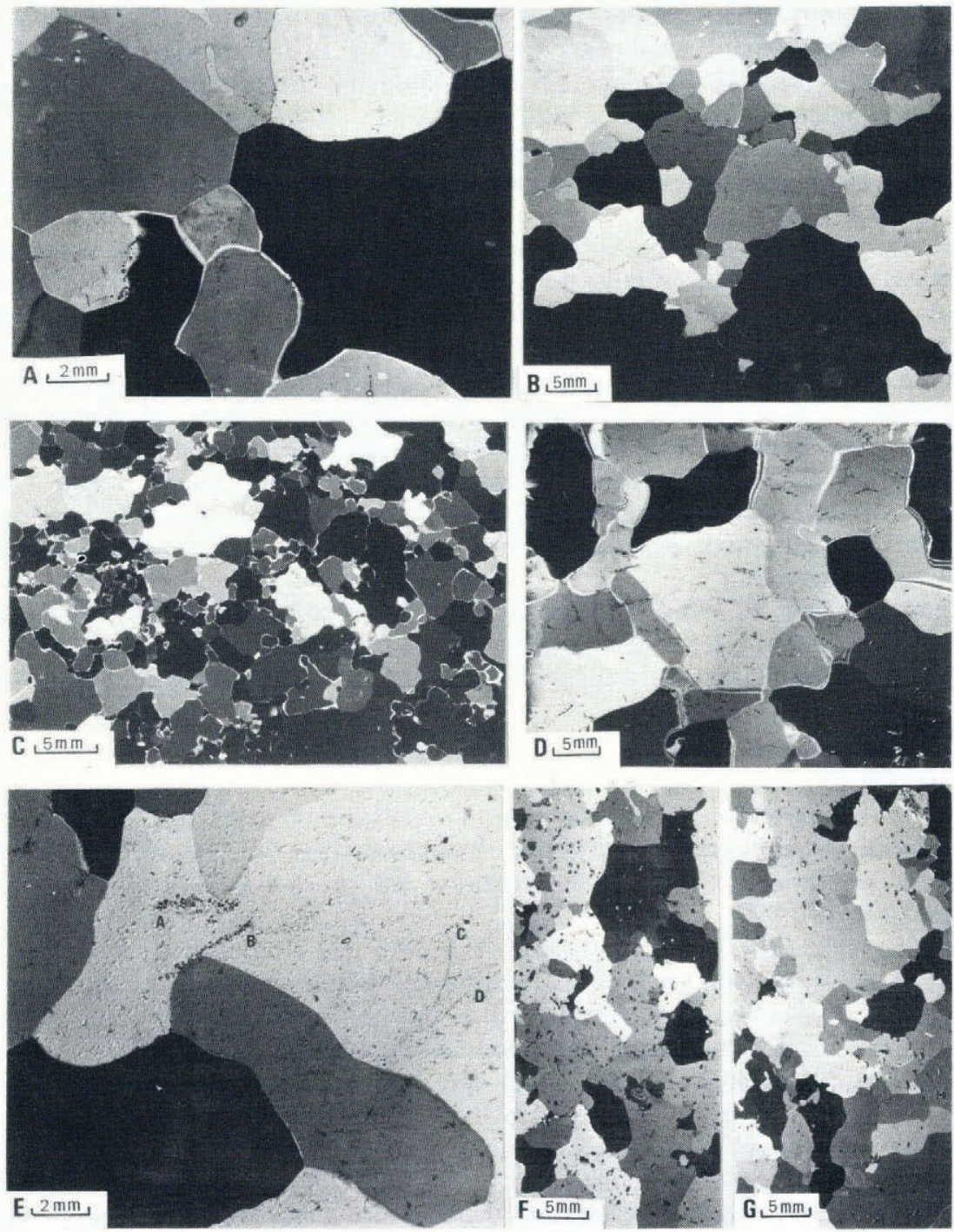

Fig. 10. Microstructures observed in the annealing experiments. A: Fine-grained starting material used in experiment 2, after $20 \mathrm{~d}$ at $1^{\circ} \mathrm{C}$. B: Annealing experiment 2 after $17 \mathrm{~d}$. C: Annealing experiment 3 after $17 \mathrm{~d}$. D: Annealing experiment 4 after $42 \mathrm{~d}$. E: Air-bubble concentrations at $A$ to $D$ in annealing experiment 4 are independent of grain boundaries and are contained within grains. F: Annealing experiment 8 after 15 d. G: Annealing experiment 9 after 39 .

There was a nucleation of new grains between shortenings of 2 and $9 \%$ in the fine- and 7 and $19 \%$ in the medium-grained samples. During the nucleation stage the total number of grains increased and consequently the average grain diameter $\bar{d}$ decreased. The processes involved in the nucleation of these small new equi-axed grains could not be identified. At these small strains there appears to be little orientation change between host and the new grain, which suggests that nucleation may be through dynamic recovery or recrystallization processes (McQueen and Jonas, 1975). To distinguish optically between a subgrain or recrystallization nucleus was not possible. Therefore, for the remainder of the paper these will be termed "recrystallized grains". 
Whether or not a critical strain for the start of recrystallization exists could not be established. However, the distribution of recrystallization nuclei in the fine-grained samples is irregular, suggesting that recrystallization is developed at localized sites. As the observed density of nuclei is higher near grain boundaries, in deformation bands, and in the more deformed regions (e.g. experiments 6 and 7), recrystallization may be associated with locally higher strains. The effect of deformation is greater the smaller the initial grain-size and the grain-size reduction during recrystallization is also notably larger.

With shortenings of $\geqslant 9 \%$ and $\geqslant 20 \%$ in the fine- and medium-grained samples respectively, there were marked increases in grain-size with no evidence for the development of additional recrystallization nuclei. The equiaxed microstructure of the recrystallized grains was destroyed by the development of irregular curved boundaries and an interlocking grain strucuture. This stage of microstructural development corresponds to a marked stage of grain growth, where the total number of grains in the deformed aggregate decreases. The growth process appears to be irregular but may in fact have been related to the rate of nucleation and mobility of the recrystallized grains. The initial grain-size and the volume fraction of recrystallized grains in the fine- versus medium-grained aggregates may in turn influence the flow stress for the aggregate. For instance grain boundaries in the fine-grained material may act so as to impede the propagation of intra-crystalline slip and influence nucleation rates throughout the ice aggregate. This in turn will influence the rate of deformation and hence the effective reduction of stress. The stress reduction of $\geqslant 12 \%$ (in the fine-grained ice) and $\geqslant 20 \%$ (in the medium-grained ice) may in fact be a contributing factor to this grain growth. The nuclei for the growing grains would presumably exist in the deformed aggregate. The irregular shapes of the coarsening grains and their rapid growth to an interlocking aggregate could correspond to the phenomenon called sometimes abnormal or discontinuous grain growth or secondary recrystallization.

In the annealing experiments, because of the varying annealing times experienced by different samples, no firm relationship could be established as to the role of deformation, and hence stored energy, on microstructural development. It is evident that the pre-existing microstructures are completely destroyed. It is believed that the existing grains prior to annealing acted as nuclei for the abnormally large grains. There is no evidence that the distribution of air bubbles inhibited coarsening; instead there was a redistribution of the bubbles.

\section{Preferred-orientation development}

In the least-deformed plane-strain specimens there is usually a rather diffuse spread of orientations, both in the host and recrystallized grain components. If measurements of recrystallized grains are separated from those of old deformed grains, there is no obvious pattern of $c$-axis preferred orientation. During the grain growth occurring at higher strains there is a development of the two concentrations in a 20 to $45^{\circ}$ small-circle about the shortening axis at the expense of the diffuse spread. This is a continuous process that appears to be intimately associated with the recovery, recrystallization, and grain growth and that yields a steady-state preferred orientation pattern. The disappearance of the diffuse spread during the recrystallization and grain-growth stages may be due to its consumption by the growth of the other grains and/or the rotation of grains. There is no real evidence that the initial grain-size enhances a particular orientation development by increasing the homogeneity of strain, although the strain-rates may be faster. Similarly there was no obvious microstructural evidence in these experiments to suggest that particular recrystallized grains in the least-deformed ice grew with the new pattern of preferred orientation, or were nucleated first and therefore have the longest time for growth in the deformed ice matrix.

The coarsening associated with the unstressed annealing experiments results in a retention of all the unannealed $c$-axis patterns with no apparent reduction in their sharpness, but instead there is a suggestion of strengthening. 


\section{CONCLUSIONS}

A set of sequential plane-strain experiments in ice at $-1{ }^{\circ} \mathrm{C}$ under a constant load suggest that a steady-state $c$-axis preferred orientation begins to develop after shortenings of $>9 \%$. This is a double maximum contained in a small-circle lying about the shortening direction. The following general conclusions have been reached:

1. This preferred-orientation pattern is enhanced during progressive deformation.

2. Preferred-orientation development is associated first with grain-reduction mechanisms which involve solid-state processes of dynamic recovery and recrystallization.

3. Once recrystallization nuclei are established in the ice aggregate, subsequent grain growth results in a rapid intensification of the preferred orientation pattern.

4. The deformation and recrystallization structures, grain-growth features and preferred orientations are developed more rapidly in the finer-grained ice aggregate.

5. The preferred-orientation patterns developed during these plane-strain deformation experiments are not substantially changed by unstressed annealing of the deformed samples.

\section{ACKNOWLEDGEMENTS}

This work was supported by a grant from the Australian Research Grants Committee. The Australian Antarctic Division provided cold-laboratory facilities for examination and preparation of the ice samples. The authors have benefited by W. F. Budd's continuous interest in and discussion of different aspects of the work.

MS. received 27 March 1980 and in revised form 8 July 1980

\section{REFERENCES}

Budd, W. F., and Matsuda, M. 1974. Nijiku-kuriipu asshuku ni okeru takesshōhyō no sentaku-hōisei ni tsuite |On preferred orientation of polycrystalline ice by biaxial creep]. Teion-kagaku: Low Temperature Science, Ser. A, [No.] 32, p. 261-65.

Exner, H. E. 1972. Analysis of grain- and particle-size distributions in metallic materials. International Metallurgical Reviews, Vol. 17, No. 1, p. 25-42.

Gold, L. W. 1963. Deformation mechanisms in ice. (In Kingery, W. D., ed. Ice and snow; properties, processes, and applications: proceedings of a conference held at the Massachusetts Institute of Technology, February 12-16, 1962. Cambridge, Mass., M.I.T. Press, p. 8-27.)

Kamb, W. B. 1972. Experimental recrystallization of ice under stress. (In Heard, H. C., and others, ed. Flow and fracture of rocks, edited by H. C. Heard, I. Y. Borg, N. L. Carter, and C. B. Raleigh. Washington, D.C., American Geophysical Union, p. 211-41. (Geophysical Monograph 16.))

McQueen, H. J., and Jonas, J. J. 1975. Recovery and recrystallization during high temperature deformation. (In Arsenault, R. J., ed. Plastic deformation of materials. New York, etc., Academic Press, p. 393-493. (Treatise on Materials Science and Technology, Vol. 6.))

Pickering, F. B. 1976. The basis of quantitative metallography. Whetstone, Metals and Metallurgy Trust for the Institute of Metallurgical Technicians.

Rigsby, G. P. 1960. Crystal orientation in glaciers and experimentally deformed ice. Journal of Glaciology, Vol. 3, No. 27 , p. 589-606.

Steinemann, S. 1954. Results of preliminary experiments on the plasticity of ice crystals. Journal of Glaciology, Vol. 2, No. 16, p. 404-12.

Steinemann, S. 1958. Experimentelle Untersuchungen zur Plastizität von Eis. Beiträge zur Geologie der Schweiz. Geotechnische Serie. Hydrologie, Nr. 10.

Wakahama, G. 1962. Köri no sosei henkei ni tsuite. I [On the plastic deformation of ice. I]. Teion-kagaku: Low Temperature Science, Ser. A, [No.] 20, p. 57-75.

Wakahama, G. 1964. Köri no sosei henkei ni tsuite. V [On the plastic deformation of ice. V]. Teion-kagaku: Low Temperature Science, Ser. A, [No.] 22, p. 1-24.

Wilson, C. J. L. 1973. The prograde microfabric in a deformed quartzite sequence, Mount Isa, Australia. Tectonophysics, Vol. 19, No. 1, p. 39-81. 